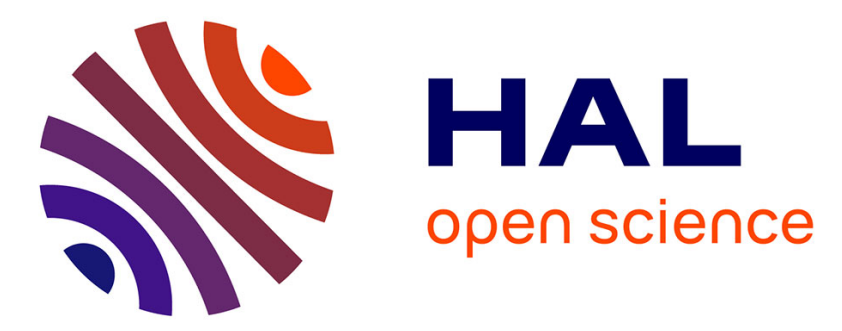

\title{
Experimental validation testing of a paired-particle matter/anti-matter propulsion system, with proposed project management
}

\author{
Mark Pickrell
}

\section{- To cite this version:}

Mark Pickrell. Experimental validation testing of a paired-particle matter/anti-matter propulsion system, with proposed project management. 2021 American Institute of Aeronautics and Astronautics Propulsion and Energy Forum, Aug 2021, Denver, Colorado, United States. hal-03477955

\section{HAL Id: hal-03477955 \\ https://hal.science/hal-03477955}

Submitted on 13 Dec 2021

HAL is a multi-disciplinary open access archive for the deposit and dissemination of scientific research documents, whether they are published or not. The documents may come from teaching and research institutions in France or abroad, or from public or private research centers.
L'archive ouverte pluridisciplinaire HAL, est destinée au dépôt et à la diffusion de documents scientifiques de niveau recherche, publiés ou non, émanant des établissements d'enseignement et de recherche français ou étrangers, des laboratoires publics ou privés. 


\title{
Experimental Validation Testing of a Paired- Particle Matter/Anti-Matter Propulsion System, with Proposed Project Management
}

\author{
M. Pickrell ${ }^{1}$ \\ Virtuoso Surgical, Inc. \\ Nashville, Tennessee 37205, USA \\ mark.pickrell@pickrell.net
}

\begin{abstract}
I. Abstract
This paper outlines experimental steps and proposed project management for experimental validation of the feasibility of a paired-particle matter/anti-matter propulsion system. The purpose of the experiments is to determine the practical feasibility of a matter/anti-matter propulsion system for generating relativistic speeds in space. The experiments are intended to determine the practicability of the four main components of a paired-particle matter/anti-matter propulsion system: the paired-particle generator, the storage system, the annihilation chamber, and the power source. Experiments will also have to be conducted regarding magnetic systems for controlling and directing the paired particles generated by the paired-particle generator through the entire system. With regard to potential project management, a mechanism for a large-scale directed-research project within the United States is considered.
\end{abstract}

\section{Background}

\section{A. Scientific Principles \& Significance}

The theoretical possibility of creating a feasible system for generating relativistic speeds (i.e., speeds at a significant fraction of the speed of light) in space, using pairs of electrons and positrons and controlling their annihilation, was proposed by the author in 2020. ["Feasibility of a matter/anti-matter propulsion system for generating relativistic speeds in space," J. Space Explor. 9(2): 162 (2020).] $]^{2}$

The basic idea of the system is that pairs of electrons and positrons can be generated in large quantities by a highenergy laser, directed toward a gold or other substrate. The electron/positron pairs can then be directed separately to a nozzle, where they interact and annihilate. When the electron/positron pairs annihilate, they release two 511-keV gamma rays, generating thrust in the nozzle by the Compton Effect. In theory, such a system is capable of generating relativistic speeds in space.

Being able to travel at relativistic speeds is extremely important. With this technology, if it is practicable, humans will be able to send probes, and even manned spacecraft, to the outer planets and nearby stars, within a single human lifetime. It is notable that two of our nearest neighboring stars are quite similar to our sun; matter/anti-matter technology may make it possible for humans to explore star systems that, potentially, are much like our own.

\footnotetext{
${ }^{1}$ General Counsel \& Chief Administrative Officer (medical-device R\&D company); 5701 Old Harding Pike, Suite 200, Nashville, TN 37205; Adjunct Professor of Law, Vanderbilt University; AIAA Member.

${ }^{2}$ See also, M. Pickrell, Humanity's Greatest Leap: The Science and Technology Behind Feasible Interstellar Space Travel, Mustang Publishing (2021), available from the publisher (hardcover - www.mustangtn.pub) and on Amazon (ebook).
} 


\section{B. Large-Scale Scientific Projects}

The directed research necessary to evaluate the practicability of matter/anti-matter propulsion is a monumental undertaking. For purposes of this discussion, "directed research" is theoretical and experimental scientific work that is aimed at evaluating the possibilities for, and achieving, a defined, practical goal. ${ }^{3}$ Directed research is the scientific work that must be performed after a concept is proposed and before prototype engineering can begin.

The two models that best illustrate large-scale directed research, comparable to the task of developing a matter/anti-matter space propulsion system, are probably the Manhattan Project and the Soviet and American space programs.

\section{The Manhattan Project}

The Manhattan Project was undertaken by the United States and some of its wartime allies (primarily Britain) during World War II to develop an atomic bomb. ${ }^{4}$ The natural occurrence of fissionable atoms was first discovered on the eve of that war, in 1938, by Otto Hahn in Nazi Germany. The existence of naturally occurring atomic fission, and the possibility that atomic fission could be induced and controlled to generate an extraordinarily powerful explosion, frightened scientific and political leadership in America and its allies. Fearing that Nazi Germany would develop an atomic weapon first, the allies (particularly the United States) committed extraordinary material resources to the task of developing an atomic weapon. As it turned out, Germany did not commit comparable resources to the development of an atomic bomb; the United States deployed the first two bombs (one made with uranium and one with plutonium) to end the war with Japan after the war in Europe was concluded by conventional means.

Much of the experimental work that was performed in the Manhattan Project involved the scientific experiments necessary to determine the possibility of, and requirements for, generating an explosive fission chain reaction. Most of this work was performed in Los Alamos, New Mexico, and this phase of the Manhattan Project was quintessential "directed scientific research." Directed scientific research, in contrast to basic science, involves the conduct of scientific experiments for the purpose of achieving a desired, practical end. In Los Alamos, experiments for evaluating the requirements for an explosive chain reaction of U-235 and Pu-239 were performed, ultimately resulting in the test of the plutonium device at Alamogordo, New Mexico in 1945. The detonation of the atomic bomb over Hiroshima, a uranium bomb, occurred without any prior test detonations, primarily because the directed research at Los Alamos had indicated that a chain reaction could be successfully initiated using U-235, with a high degree of certainty, and because of the difficulty in separating U-235.

The physical infrastructure necessary for the production of plutonium and uranium in adequate quantities required massive construction - in Chicago; Los Alamos; Oak Ridge, Tennessee; and Hanover, Washington. The project was managed by the United States War Department using many civilian contractors.

\section{The U.S. Space Program}

The technology for chemical rockets, particularly ones capable of generating orbital speeds, developed more slowly than the technology for achieving explosive fission devices. Robert Goddard used a liquid-fueled rocket for his high-altitude rocket in the 1920s, and the Germans in World War II used much larger liquid-fueled engines to power their $\mathrm{V}-2$ rockets.

The U.S. Space Program was similar in many ways to the Manhattan Project. ${ }^{5}$ Though not undertaken in the midst of a shooting war, the Mercury and Apollo programs were undertaken in the midst of the Cold War. There were certainly military aspects of the Soviet Union's and the United States' space programs, because a rocket that can put

\footnotetext{
${ }^{3}$ See, e.g., Energy Research and Development Administration Act, Pub. L. 95-238 (1977) (establishing Laboratory Directed Research and Development Program consistent with the Atomic Energy Act, 42 U.S.C. § 2011, et seq (1954)).

${ }^{4}$ See generally, L. Groves, Now It Can Be Told: The Story of the Manhattan Project, Da Capo Press, New York, 1962 .

${ }^{5}$ See generally, W. MacDougall, ...the Heavens and Earth: A Political History of the Space Age, The Johns Hopkins University Press, Baltimore, 1985.
} 
a manned space capsule into orbit around the earth was also capable - and visibly so - of delivering a thermonuclear device anywhere on the Earth. Military purposes aside, there were considered to be political, cultural, and scientific benefits to developing orbital capability and, from the perspective of the United States, the capability of ultimately sending manned spacecraft to the Moon.

In response to the Soviet launch of the Sputnik satellite, the Eisenhower Administration established NASA in 1958. As envisioned, NASA would be a civilian agency created for, among other things, "The improvement of the usefulness, performance, speed, safety, and efficiency of aeronautical and space vehicles." 6

President Kennedy famously focused the mission of the U.S. Space Program in a speech at Rice University on September 12, 1962, by declaring that manned exploration of the Moon, by the end of the decade, was the program's primary goal.

Similar to the directed research of the Manhattan Project, the directed research that was necessary for successful launch of manned spacecraft to the Moon was extraordinarily expensive and complex. While some of the technology from Germany's V-2 program had been developed in the United States since the end of World War II, there was still tremendous directed research that had to be performed by the United States in order to achieve orbital speeds, and then to achieve escape velocities with manned spacecraft.

\section{U.S. National Laboratories}

With the end of World War II, the Manhattan Project itself was terminated. The goal of being the first combatant to gain atomic capability had been achieved. But the facilities at the heart of the Manhattan Project were not mothballed. National laboratories in Oak Ridge and Los Alamos, as well as Sandia National Laboratory in New Mexico, continued to conduct scientific research, often conducting basic scientific research but also conducting directed research (particularly with regard to nuclear physics and nuclear weaponry). Brookhaven National Laboratory was founded in 1947, the Princeton Plasma Physics Laboratory was founded in 1951 (starting as Project Matterhorn), Lawrence Livermore National Laboratory was founded in 1952, and FermiLab was established in 1967. They similarly have conducted basic research, while also conducting directed research (again, particularly with regard to nuclear physics and nuclear weaponry).

The U.S. national laboratories possessed, and still possess, the capability of conducting directed research, in addition to performing significant basic research. While the United States funds much scientific work, including directed research, through universities (often funded through the National Science Foundation, the National Institutes of Health, and DARPA, for example), the national laboratories still possess the land, buildings, equipment, technical knowledge, and command-and-control administration for conducting directed scientific research on a large scale.

${ }^{6}$ The Space Act, Pub. L. 85-568, 72 Stat. $426 \S 102$ (1958). 


\section{Validation Experiments}

To determine the practicability of matter/anti-matter annihilation for generating relativistic speeds in space, significant directed research must be performed to determine the requirements, and optimized capabilities, of the major components of a matter/anti-matter propulsion system. Those components likely are: 1) a paired-particle generator; 2) a storage system, such as twin tokamaks; 3 ) a nozzle/annihilation chamber; 4) a power source (most likely, a fissionbased nuclear reactor); and 5) systems for conveying the paired particles from the generator through the rest of the system, to annihilation. The major testing, evaluation, and validation for each of these components is described below.

\section{A. Paired-Particle Generator}

The requirements, limitations, and capabilities for the paired-particle generator must be determined by experimental research. As discovered by Hui Chen and her team at Lawrence Livermore National Laboratory, a highenergy laser directed at a gold substrate generates large quantities of pairs of electrons and positrons.

In order to advance matter/anti-matter propulsion technology, the following, at a minimum, must be established experimentally:

1. The best medium, wattages, optics, and beam concentration of a high-energy laser for the purpose of generating large quantities of electron/positron pairs;

2.The best metallic substrate for the purpose of generating large quantities of electron/positron pairs;

3. The quantities of electron/positron pairs that can be generated by an optimized laser and metallic substrate;

4. Any degradation, or the amount of degradation, of the metallic substrate, when used for the purpose of generating large quantities of electron/positron pairs; and

5. The expected useful life of an optimized laser, when used for the purpose of generating large quantities of electron/positron pairs.

\section{B. Annihilation Chamber}

The requirements, limitations, and capabilities of the annihilation chamber must be determined by experimental research.

In order to advance matter/anti-matter propulsion technology, the following, at a minimum, must be established:

1. The optimal size and shape of the annihilation chamber (to avoid electric-charge buildup and to maximize thrust from gamma rays generated in the annihilation chamber);

2. The optimal material for construction of the interior lining of the annihilation chamber (to avoid electriccharge buildup and to maximize thrust);

3. The amount of radiation shielding that will be necessitated by the ionizing radiation generated in the chamber; and

4. The amount of thrust that can be generated from an optimized system.

It is in the development and testing of the annihilation chamber that the success or failure of this system is most likely to be revealed. The nature of the energy transfer from the gamma rays to the atoms of the lining of the annihilation chamber is crucial. If the energy transfer is translational, then this technology will likely be successful. If the energy transfer is not translational (i.e., electrical, or somehow cancelling out), then this technology will likely be unsuccessful as a means for space propulsion. 


\section{Storage}

The requirements, limitations, and capabilities for the paired-particle storage system, if desired or advisable in light of the results of the experimentation regarding the paired-particle generator, must be determined by experimental research. As discovered by Eve Stenson and her team at the Max Planck Institute for Plasma Physics, paired particles can be stored in a dipole stellarator for a significant period of time. Depending on the expected mission, and depending on the capabilities of the paired-particle generator, a storage system, such as twinned tokamaks, may be desirable for an optimized matter/anti-matter propulsion system.

In order to advance matter/anti-matter propulsion technology, the following may need to be determined experimentally:

1. The optimal conformation of a paired-particle storage system;

2. If twin tokamaks are the optimal conformation of a paired-particle storage system, the optimal size, weight, magnets, and magnetic fields, for storage of electron/positron pairs; and

3. The potential capacity of a paired-particle storage system.

Based upon the results of the experiments related to paired-particle generation, it will be possible to determine whether a storage system is even required for a given mission. It may be possible and advantageous (in weight-saving alone) to direct the paired particles from the generator to the annihilation chamber without any storage necessary.

\section{Energy Source}

A matter/anti-matter propulsion system is likely to require a fission-based nuclear reactor as the source of its power, for propulsion, control systems, and any necessary life support. The following, at a minimum, must be determined:

1. The optimal fuel for the reactor; and

2. The optimal materials and weight of the reactor, in light of: energy requirements of the system, safety requirements of the system, and any necessary radiation shielding.

\section{E. Electron \& Positron Conveyance}

Hui Chen and her team have demonstrated that electrons and positrons generated in the paired-particle generator can be separated magnetically. Once paired particles are generated, they will have to be controlled and directed, either directly to the annihilation chamber or to and from a storage system. Therefore, assuming that a storage system is necessary for a given mission, the following, at a minimum, will have to be determined experimentally:

1. The safest and most efficient conformation for separating and directing electrons and positrons;

2. Assuming that the safest and most efficient conformation for transporting electrons and positrons is a column of magnets, the optimal magnets and magnetic fields for the columns, as well as the optimal size and curvature of the column; and

3. The safest and most efficient transmission mechanism for conveying charged particles from one component of the system to another: from the paired-particle generator to a magnetic column, from a magnetic column to a storage system; or from a storage system or a column to the annihilation chamber. 


\section{Project Management \& Execution}

If the United States, or another nation, or a consortium of nations, decides to undertake the scientific research necessary to advance matter/anti-matter propulsion, management of a project of this magnitude will be extremely difficult and expensive. The planning, budgeting, funding, purchasing, construction, execution, and coordination of the experimental phase of this project, alone, is an extraordinary task. To say nothing of the costs and coordination, if the experimental phase is promising, of the follow-on prototype development, fabrication, and testing.

If the United States were to undertake the development of matter/anti-matter technology for space propulsion, NASA is the logical lead agency. By statute, the development of technologies like this has been their mandate since the agency was established.

The national laboratories of the United States also appear to be perfectly suited to conduct the necessary directed research. Because the most important experimental work, to date, with paired-particle generation has been conducted at Lawrence Livermore, that laboratory is an obvious organization to conduct the necessary experiments for optimizing a paired-particle generator. Because of its work on fusion tokamaks, the Princeton Plasma Physics Laboratory is an obvious candidate for conducting experiments on the storage system. Because of its work on nuclear-reactor design, particularly work on lightweight nuclear reactors (for possible use by the U.S. Air Force), Oak Ridge National Laboratory may be well-suited for research regarding the power source for the system. The National High Magnetic Field Laboratory, funded by the National Science Foundation and other organizations, may be well-suited to evaluate potential transmission mechanisms for safely conveying large quantities of electrons and positrons from one component of the system to another.

If a political commitment is established in the United States for supporting the development of matter/anti-matter propulsion, the planning and preparation for the experimental phase of this project can be properly conducted. To the extent that multiple nations decide to collaborate on the development of this technology, overall management and division of responsibilities can be worked out by mutual agreement. There may even be a benefit to having separate efforts geared toward tackling the same experimental questions, within the United States or internationally.

\section{Conclusion}

In theory, based upon the laboratory discoveries of Chen and her team at Lawrence Livermore, and Stenson and her team at Max Planck, matter/anti-matter annihilation as a source of energy for generating relativistic speeds in space is conceivable. If development of this technology is successful, humans will be able to send probes, and even manned spacecraft, to the outer planets and nearby stars within a single generation.

In order to determine the practical feasibility of matter/anti-matter propulsion, significant directed research must be conducted. The experimental phase necessary for furthering this technology will require experimentation and testing on the likely necessary components of a matter/anti-matter system: paired-particle generation, paired-particle conveyance, storage of paired particles, controlled annihilation of paired particles, and an appropriate energy source for the entire system.

In the United States, NASA is the obvious governmental agency for overall management of the experimental phase of this development project (as well, eventually, as the prototype and operational phases of this project). The United States' national laboratories are also likely candidates for performing some, or most, of the component experimentation and testing for the initial experimental phase of this important project.

\section{Acknowledgements}

Some of the ideas presented in this paper were first presented, in nascent form, in an oral presentation to the Annual Technology Seminar of the Houston Chapter of the AIAA, in October 2020. The invitation to present matter/anti-matter technology to that group, and the substantive comments from the attendees in response to the presentation, are greatly appreciated. Similarly, the invitation to present this paper at the 2021 AIAA Propulsion \& Energy Forum is greatly appreciated. Particularly for someone of my background, I greatly appreciate the willingness of scientists, engineers, and skilled administrators to seriously consider my hypothesis. 